\title{
INFLUENCIA DE LA CIUDAD COLONIAL HISPANOAMERICANA EN EL ENSANCHE DE BARCELONA Análisis morfológico del Plan Cerdà para Barcelona de 1859
}

\author{
Influence of the hispanoamerican colonial city in \\ the Barcelona Ensanche \\ Morphological analysis of the Cerdà Plan for Barcelona of 1859
}

\author{
Malave Fernández, Rafael Alirio; \\ Universidad de Las Américas, Universidade da Coruña \\ rafael.malave@udc.es \\ Aceves Álvarez, Oscar;
}

\section{RESUMEN}

El Ensanche de Barcelona, proyectado por el ingeniero de caminos Ildefons Cerdà en 1859, es un ejemplo notable del urbanismo del siglo XIX, cuya influencia ha llegado a otros ensanches posteriores gracias a su calidad y al avanzado pensamiento que lo generó. A pesar de la originalidad de muchos aspectos del llamado "Plan Cerdà", este se encuentra relacionado con algunos precedentes, siendo la influencia de la ciudad colonial hispanoamericana, concretamente la del "modelo clásico" -de trazado urbano cuadricular-, la que nos interesa analizar aquí. ¿De qué manera entonces tomó Cerdà como referencia las ciudades hispanoamericanas para diseñar su propuesta para el ensanche de Barcelona? Bajo la hipótesis de que la influencia es explicita en lo que se refiere a forma, orden, ocupación del espacio, y capacidad de expansión, e implícita -pero evidente- en cuanto al ancho de las calles y el tamaño de las manzanas, el objetivo principal de la investigación es exponer evidencias que hacen verosímil la posible influencia de las ciudades hispanoamericanas en el diseño del Plan Cerdà.

Palabras clave: Cerdà, Morfología, Hispanoamérica, Barcelona, Ensanche.

Bloque temático: Teoría e historia de la ciudad.

\begin{abstract}
The Barcelona's Extension, designed by the road engineer Ildefons Cerdà in 1859, is a notable example of nineteenth-century urbanism, whose influence has reached other subsequent city extensions thanks to its quality and the advanced thinking that generated it. Despite the originality of many aspects of the so-called "Plan Cerdà", this is related to some precedents, being the influence of the Spanish-American colonial city, specifically that of the "classic model" (of urban grid layout), which interests us to analyze here. In what way did Cerdà take as a reference the Spanish-American cities to design his proposal for the widening of Barcelona? Under the hypothesis that the influence is explicit in terms of form, order, occupation of space, and capacity for expansion, and implicit -but evident- in terms of the width of the streets and the size of the apples, the research main objective is to expose evidence that make likely the possible influence of Spanish-American cities on the design of the Cerdà Plan.
\end{abstract}

Keywords: Cerdà, Morphology, Hispanic America, Barcelona, Ensanche.

Topic: Theory and history of the city. 


\section{Introducción}

Para el inicio del siglo XIX Barcelona aún seguía siendo una ciudad con fuerte morfología medieval: trazado sinuoso, perímetro amurallado y poca infraestructura para el manejo de residuos. Como consecuencia de esto, existía un caos urbano que afectaba las condiciones higiénicas de la ciudad: no había suficiente infraestructura sanitaria, los edificios excedían sus límites de altura y estaban apiñados entre sí contando con pocos espacios abiertos en su interior; lo que provocaba falta de ventilación, de agua limpia, insalubridad e incluso el brote de epidemias (El Haddad, 2017). Sumado a esto, tanto el aumento de la migración campo-ciudad y por consecuencia el incremento de la población, como la conformación de una nueva burguesía y el desarrollo industrial terminaron por detonar la crisis urbana que obligó a tomar medidas. A pesar de que en 1859 el Ayuntamiento de Barcelona convocó a un concurso para el diseño del denominado "Distrito del Ensanche" -del cual resultó ganador Antonio Rovira y Trías, las decisiones de la ampliación sobre el llano de Barcelona serían tomadas desde Madrid. Un año antes, por medio de una Real Orden, la ciudad condal abandonaba su condición de Plaza Fuerte y se favorecía el Anteproyecto de Ensanche desarrollado por el ingeniero de caminos, canales y puertos Ildefons Cerdà i Sunyer (1815 - 1876) desde 1855 y cuya principal característica consistía en la aplicación del Plan Hipodámico o trazado de damero complementado con una serie de vías diagonales en un área aprox. de $110 \mathrm{Ha}$.

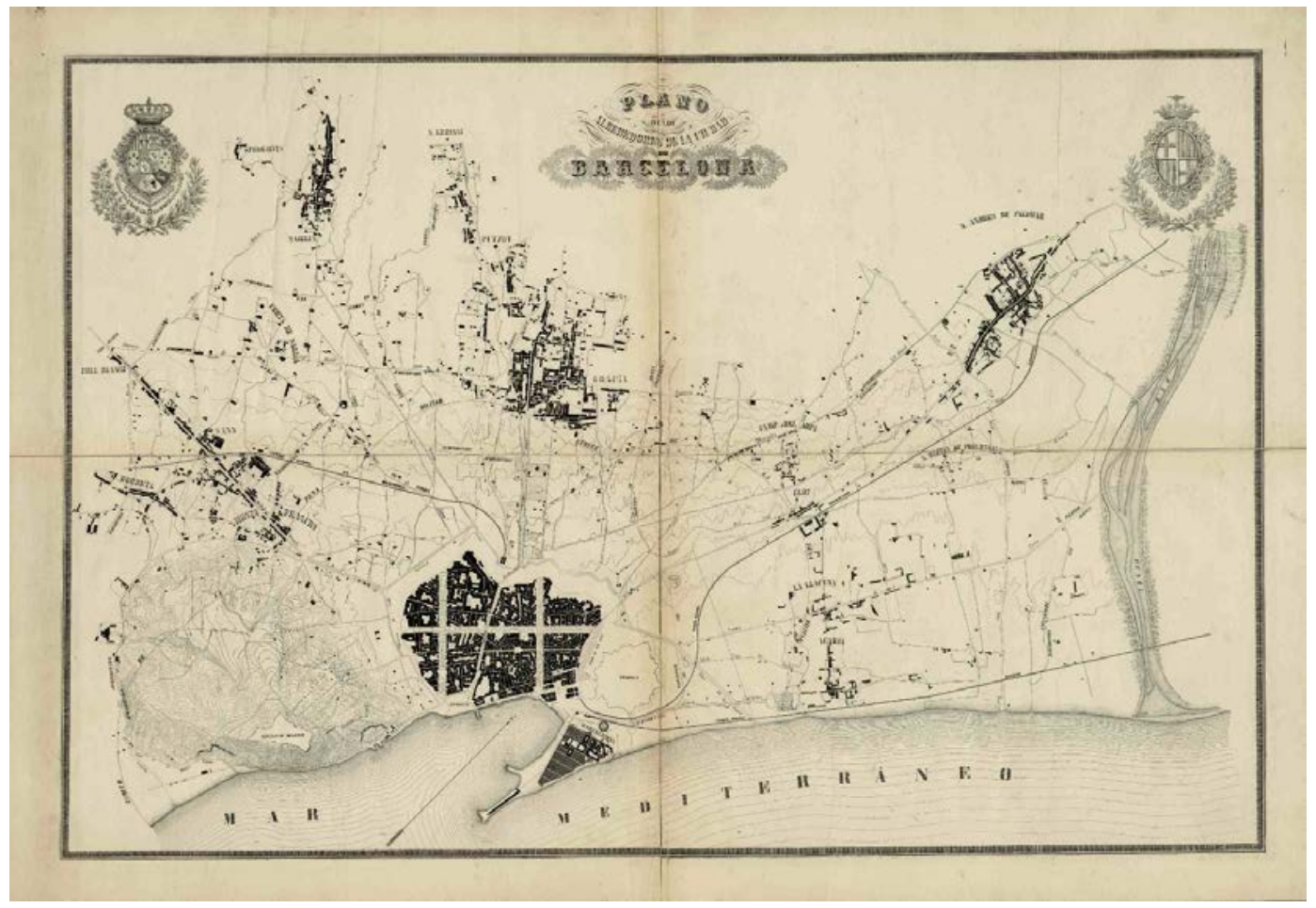

Fig. 01 Plano de Barcelona antes del Ensanche.

Fuente: Cerdà, I. (1855) Plano de los alrededores de la ciudad de Barcelona. [Plano]. Escala 1:10.000.

Recuperado de:

http://www.anycerda.org/web/imagen/1266924933.foto1.tbl_fichas.0/Topogr\%C3\%A0fic\%20de\%20Cerd\%C3\%A0\%201855.\%2012-

24_1_2\%20Baixa.jpg. Consultado 15 de Julio 2019.

El característico esquema de repetición de manzanas cuadradas establece una relación formal directa y evidente entre el ensanche y el modelo clásico -denominación de Hardoy (1972) para referirse a las ciudades coloniales hispanoamericanas con esquema de trazado cuadricular o de repetición de manzanas rectangulares iguales-, es decir, entre Barcelona y ciudades como Santiago de Chile, Montevideo o Buenos Aires, principalmente en las zonas próximas al casco histórico de estas ciudades americanas. No hace falta más que ver una fotografía aérea o mirar un plano para comprobar cierta similitud. No obstante, afirmar dicha influencia 
no tendría peso basándose solo en una lectura superficial, puesto que existen ejemplos cuadriculares en otras locaciones que pudieran o no estar emparentados, existiendo la posibilidad de ser el resultado de evoluciones convergentes. Es por tanto necesario establecer una conexión respaldada por otros criterios: la formación y contexto histórico social de Cerdà, los escritos del propio Cerdà, el Proyecto de Ensanche de Barcelona, y la realidad física comparada entre el modelo hispanoamericano de ciudad -el arquetípico cuadricular- y el modelo del Ensanche.

Es necesario aclarar que la influencia de las ciudades hispanoamericanas en el Plan Cerdà ha sido investigada y expuesta por autores como Bonet Correa (1977), y De Terán (1999) y (2009), sirviéndose ambos autores de la revisión del contexto de Cerdà y de su obra escrita para argumentar dicha influencia. No obstante, esta investigación busca aportar al conocimiento sobre el tema a partir del contraste de fuentes escritas (por Cerdà y otros autores) y fuentes gráficas (cartografía), y de la comparación morfológica entre ambos modelos.
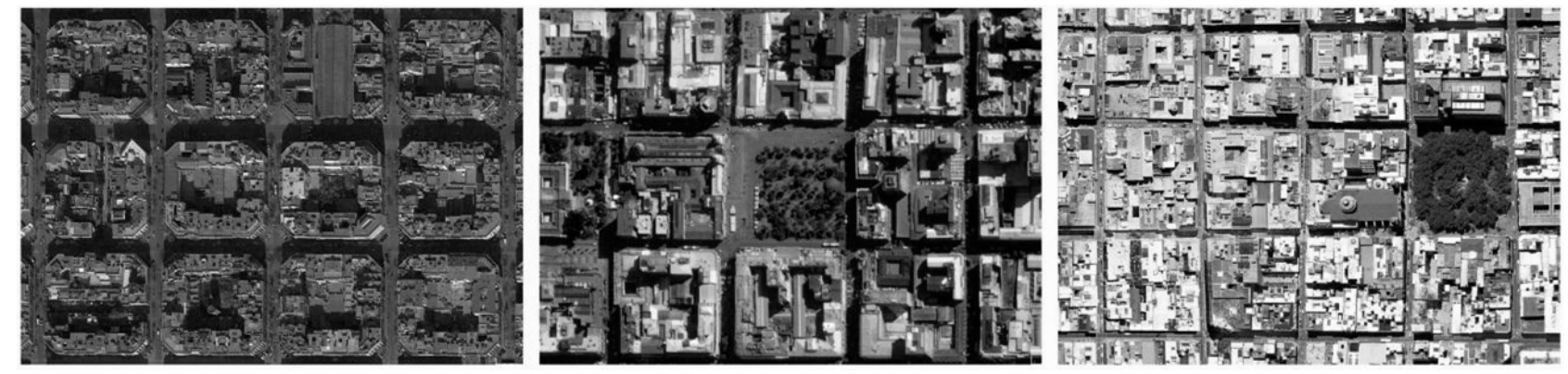

Fig. 02 Vista aérea de segmentos de Barcelona, Santiago de Chile y Montevideo. Fuente: Google Earth

\section{Urbanismo del siglo XIX}

Si bien la actitud de asumir el diseño de la ciudad a la manera del diseño de un edificio fue asumida por los arquitectos "modernos" del Primer Renacimiento del siglo XV, la aplicación de dichas ideas no pasó de algunas intervenciones puntuales en ciertos barrios de las urbes medievales. Para historiadores como Pevsner, Benévolo o Castex, las reformas urbanas desarrolladas a finales del siglo xV en ciudades como Ferrara, Urbino, o Pienza, son fiel reflejo de este cambio de paradigma, sin embargo, en el caso de Europa, los sueños renacentistas de la "ciudad ideal" se quedarían por el momento en desarrollos específicos como Palmanova ciudad italiana fortificada del siglo XVI-, o en el papel, como la Sforzinda de II Filarete. Cabe señalar que en la América Española dichas ideas si se lograron materializar a una escala importante, pues según Benévolo (1968) el esquema urbano ideado en América en las primeras décadas del 500 es el único modelo de ciudad producido por la cultura renacentista y controlado en todas sus consecuencias ejecutivas.

Volviendo al escenario europeo, es en el siglo XIX cuando las utopías urbanas planteadas durante 300 años se encuentran en un contexto social, político y económico que cuenta con la capacidad de llevarlas a cabo. El siglo inicia con una fuerte crítica a las consecuencias del desarrollo de la Revolución Industrial en el espacio urbano (Alarcón, 2002), requiriendo que las antiguas capitales imperiales europeas se adapten al aumento de población urbana, a la incorporación de mayores centros de producción, almacenaje e intercambio de productos, y a la aparición de nuevas edificaciones y espacios públicos representativos del poder republicano.

Como bien relata De Terán (1999), en este período se pueden identificar claramente dos posturas adoptadas por los profesionales de la época para la reforma y/o ampliación de grandes áreas urbanas e incluso para la construcción de nuevas ciudades; por un lado se encontraría una iniciativa "estético prestigiosa", derivada de la actitud neoclasicista e historicista que buscaría rescatar y aplicar los ideales renacentistas y barrocos enfocados en la jerarquización, la perspectiva, y la monumentalidad de las ciudades, y teniendo como elocuentes exponentes de la época los casos de la reforma de Haussmann para París, el Ringstraße de Viena o el National Mall de Washington. Por el otro lado se encontraría la postura "técnico racional": pragmática, 
ordenada y fiel representativa de la retícula ortogonal que puede apreciarse en ampliaciones $u$ ordenamientos de ciudades europeas como Edimburgo, Glasgow, o Berlín a principios del siglo XIX, o años más tarde en el caso de La Plata en Argentina. Si bien la primera era la postura más vanguardista por los profesionales de la época, en el caso de España dominaba la segunda aproximación.

Para De Terán, Cerdà se encontraría -por contexto y por demostración de su propia obra- bajo esta postura "técnico racional", y en cuanto a la influencia de las ciudades hispanoamericanas en el Proyecto de Ensanche de Barcelona defiende que existen razones históricas que hacen probable la relación entre estos dos modelos de ciudad; a) el ser la ciudad hispanoamericana producto de la misma sociedad, b) el hecho de que Cuba era aún parte de España en la época de Cerdà y aún después de la independencia de las colonias continentales se siguieron fundando ciudades en la isla -por ejemplo Cienfuegos, que presenta un trazado cuadricular y fue fundada en 1819, solo 40 años antes del proyecto de ensanche de Barcelona-, c) la experiencia y conocimiento de los ingenieros militares españoles en este campo, y d) el conocimiento que se encontraba y se encuentra almacenado en España sobre las ciudades americanas y su fundación: cartografía y documentos. A partir de estos cuatros aspectos, sumado otros, se puede inferir la probable referencia, ya que además según Bonet Correa (1977) Cerdà no era ajeno a la realidad de su tiempo y al conocimiento de las ciudades hispanoamericanas de origen colonial.

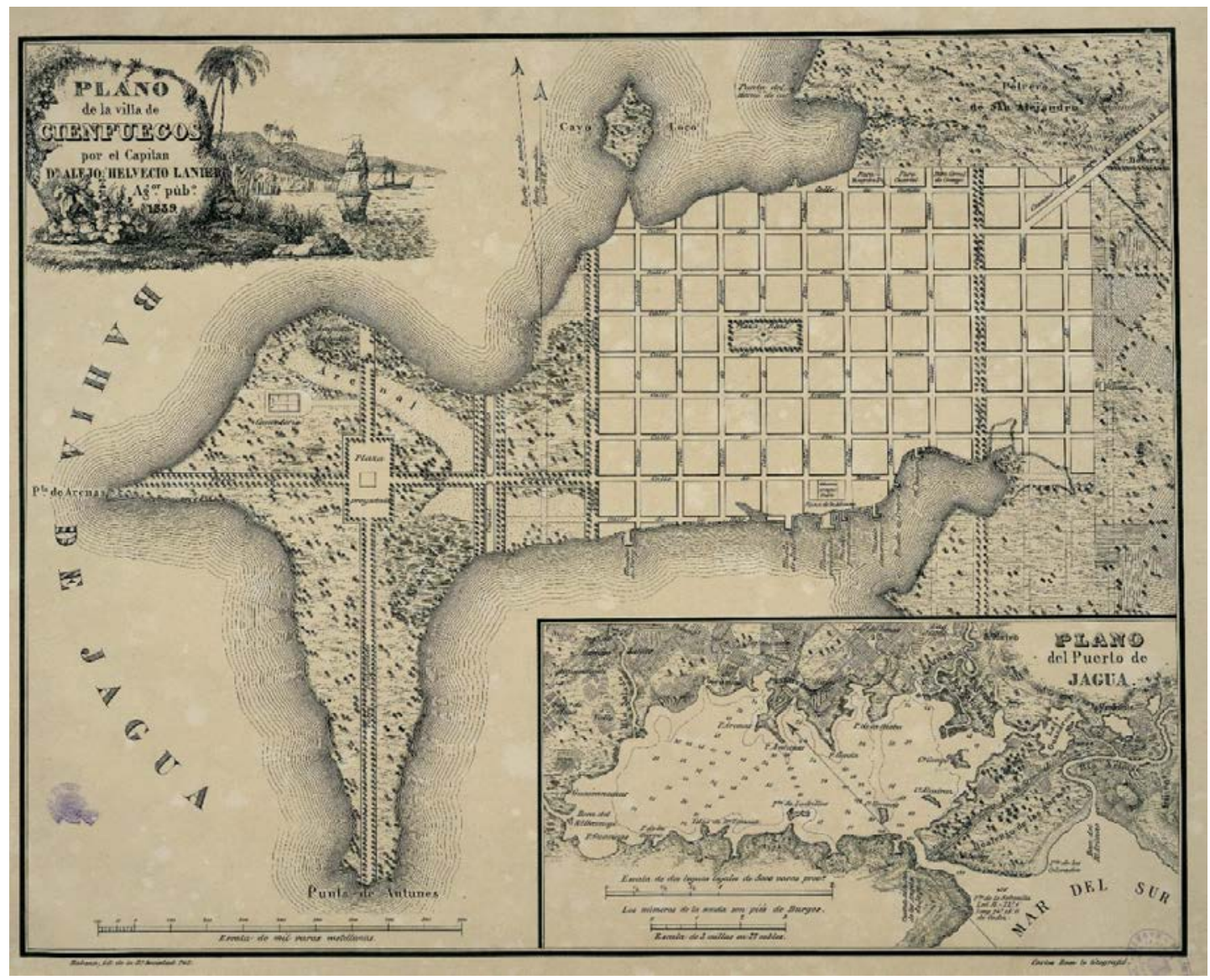

Fig. 03 Plano de Cienfuegos de 1839.

Fuente: Laniers, A. (1839) Plano de la villa de Cienfuegos. [Plano]. Escala ca. 1:84.000.

Recuperado de:

http://bdh.bne.es/bnesearch/biblioteca/Lanier,\%20Alejo\%20Helvecio;jsessionid=A5EF5AD0C5C2940F2CFEA6166D402C86.

Consultado 15 de Julio 2019. 


\section{Referencias al modelo Hispanoamericano en las obras escritas de Cerdà}

Pero más allá de la evidencia circunstancial, quizás el argumento más importante para confirmar la influencia planteada en esta investigación es la referencia explícita que realizó Cerdà sobre la ciudad colonial hispanoamericana en sus escritos, lo cual validaría por completo la relación entre ambos modelos. A partir del análisis de dos de sus obras escritas es posible, no solo constatarlo, sino también apreciar de qué manera se establece esta relación.

En la "Teoría General de la Urbanización", publicada en 1867, Cerdà hace una revisión histórica de diferentes modelos de ciudad, en el cual compara el modelo de ciudad medieval europeo (irregular) presente en España con el hispanoamericano, diciendo lo siguiente.

"Tanto es así, como que nuestra misma raza que por las razones dichas toleraba en la Península semejante monstruosidad, al encontrarse en situación holgada para poder obrar libremente á impulsos de las inspiraciones de su génio y de sus generosos instintos, fundaba en América ciudades vastísimas bajo un sistema tan acertado, que ha podido servir durante siglos enteros de tipo y modelo á muchos pueblos, aun á aquellos que de mas ilustres y cultos se precian." (Cerdà, 1867-1968: 187).

De esta manera Cerdà alaba al sistema de ciudades hispanoamericanas y también señala su influencia en asentamientos urbanos posteriores en otras regiones. Sobre la forma y estructura de estas ciudades menciona lo siguiente:

"Las calles fueron anchas y espaciosas para que pudiera servir holgadamente al movimiento urbano, no al que entonces, en la época de la fundación, pudiese existir, sino al que habria de desenvolverse con el aumento de población muy atinadamente previsto.

La isla que la red de vías urbanas dejaba á la edificación fueron ortogonales y asaz espaciosas para no imponer, ó mejor, para en lo posible prevenir las construcciones condensadas en demasia. Este hábil sistema el único verdaderamente adecuado á una buena urbanización, permitia fácilmente convertir una isla en plaza en el sitio en que la concurrencia natural lo aconsejase, sin necesidad de producir transtorno ni modificación en el resto del plan." (Cerdà, 1867-1968: 188).

Se entiende así que, además de valorar el ancho de las calles, el tamaño de las manzanas, el orden de la red viaria y la ortogonalidad, Cerdà cataloga el modelo como "el único verdaderamente adecuado a una buena urbanización" (1867-1968: 188). Por tanto, al referirse su propio autor de este manera no resulta aventurado pensar que éste modelo fuera una de las influencias para el proyecto de Ensanche de Barcelona.

Pero si aún queda alguna duda, su otra obra, la "Teoría de la construcción de ciudades aplicada al proyecto de reforma y ensanche de Barcelona" la despeja. Esta obra, aunque escrita en 1859 -antes que la "Teoría General de la Urbanización"- no se conocía hasta 1987 cuando fueron encontrados los documentos que la componen, los cuales fueron publicados en 1991. Estos escritos esclarecen muchos aspectos de cómo fue concebido el proyecto, pudiendo contrastar algunos argumentos planteados acerca del ensanche previos a la publicación de esta obra e incluso posteriores a ésta -como Solá-Morales en 1974, 1978, y de forma más matizada en 1997 -, en los cuales se defiende lo original de la propuesta y por tanto niega la relación aquí expuesta: "Frente a todos ellos, la aprobación del proyecto Cerdá, supone, en cambio, la de un proyecto que no tiene más lógica que la suya propia" (Solá-Morales 1974, p. 134).

Lo primero que esclarece este texto de Cerdà es el origen de la forma de la manzana, justificado como una manera de aprovechar los vientos para incrementar la salubridad, estableciendo también la orientación de las mismas y aludiendo lo siguiente: “...De modo que según esto el esqueleto general de las calles vendrá á ser un sistema de cuadriculos en forma de tablero de damas como se hallan dispuestas las de la mayor parte de las ciudades de America" (1991: 374-375, §1201).

Cerdà establece así una relación formal entre los dos modelos, y sella posteriormente esta referencia cuando dice: "...dese á este ensanche una forma y estension que permitan al caserio distribuirse de una manera 
analoga al de las poblaciones fundadas por nuestros ilustres abuelos en el continente americano" (1991: 407, §1462). En este sentido, no es solo una referencia formal al modelo hispanoamericano, sino una explícita declaración de intenciones de su parte.

\section{La metodología del diseño de las manzanas}

Sin embargo, Cerdà no se limita a tomar el modelo de cuadrícula y a utilizar su forma, sino que llega a él a través del análisis y la sistematización, y de estudiar algunas ciudades hispanoamericanas. Como se dijo antes, la orientación y forma ortogonal de las manzanas es justificada por él para el aprovechamiento de los vientos y establece que esta sea cuadrada.

Luego, por lo que se ve en el documento, examina las dimensiones de manzanas y anchos de calle -entre otros parámetros- de diferentes ciudades, incluyendo Lima y Buenos Aires. Indica que Lima tiene manzanas cuadradas de 110 metros de lado y calles de entre 15 y 20 metros, mientras que en Buenos Aires las manzanas tienen 116 metros de lado y las calles 20 metros de ancho. No menciona la fuente de dichas medidas.

La medida mínima que asigna a las calles del proyecto es 20 metros de ancho, justificándola como la altura que estima en los edificios, y aunque no lo expresa, está claro que la medida coincide con la de los referentes. Indica a su vez que otras calles medirán 30 metros de ancho -en circulaciones de cierta importancia-, y 50 metros en las más importantes, previendo así circulaciones intensas.

En cuanto al tamaño de la manzana, Cerdà establece que esta deberá de ser el mínimo de manzana con holgura suficiente y en relación con la altura, fondo de la parcela, ancho de la calle y habitantes por edificio. Definiendo como variables la volumetría y las aristas de las manzanas, plantea y aplica una fórmula para testear y evaluar la dimensión más eficiente a partir de 4 variaciones morfológicas: a) Manzana cerrada achaflanada, b) Manzana cerrada sin chaflanes, c) Manzana abierta achaflanada, y d) Manzana abierta sin chaflanes.

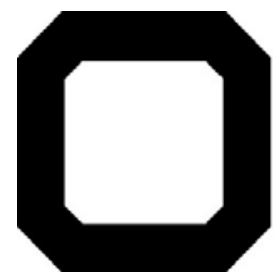

a)

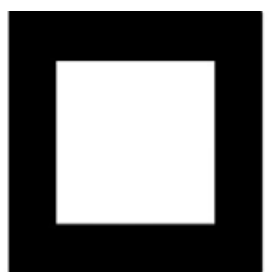

b)

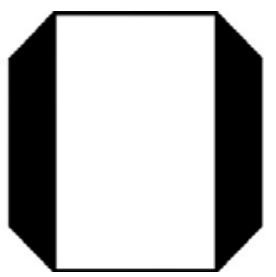

c)

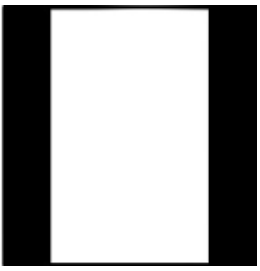

d)

Fig. 04 Esquemas de 4 variaciones morfológicas de manzana. Fuente: Elaboración propia. 
La fórmula planteada por Cerdà es la siguiente:
x...... Lado de la manzana
2b... Anchura de la calle
f...... Fondo del solar de construcción
d...... Fachada del solar de construcción
V...... Número de habitantes por casa
p..... Número de metros de superficie que han de tocar por individuo de la total de la población

Cerradas $\left\{\begin{array}{c}\text { Con chaflanes } x=\frac{2 p v-2 b d}{d} \pm \sqrt{\frac{4 p v\left(p v f-2 b d f-b^{2} d-d f^{2}\right)}{d^{2} f}} \\ \text { Sin chaflanes } x=\frac{2 p v-2 b d}{d} \pm \sqrt{\frac{4 p v(p v-2 b d-d f)}{d^{2}}}\end{array}\right.$

Abiertas $\left\{\begin{array}{c}\text { Con chaflanes } x=\frac{p v-2 b d}{d} \pm \sqrt{\frac{p v\left(p v f-4 b d f-4 b^{2} d\right)}{d^{2} f}} \\ \text { Sin chaflanes } x=\frac{p v-2 b d}{d} \pm \sqrt{\frac{p v(p v-4 b d)}{d^{2}}}\end{array}\right.$

Aplicando ahora a estas fórmulas los siguientes valores constantes:

$\begin{array}{lr}2 b=20 & Y \text { suponiendo además que los valores de } V \text { sean } \\ f=20 & 57 \\ d=20 & 43 \\ p=40 & 29\end{array}$

(Cerdà, 1991: 413, §1497).

Al aplicar los 4 factores diferentes para la variable de habitantes en el cálculo de las 4 variaciones planteadas, se obtienen los siguientes resultados: 
a) Manzanas cerradas achaflanadas:

a.1) 71 hab. $=498,72 \mathrm{~m}$. a.2) 57 hab. $=385,38 \mathrm{~m}$. a.3) 43 hab.= 270,76 m. a.4) 29 hab.= 150,92 $\mathrm{m}$

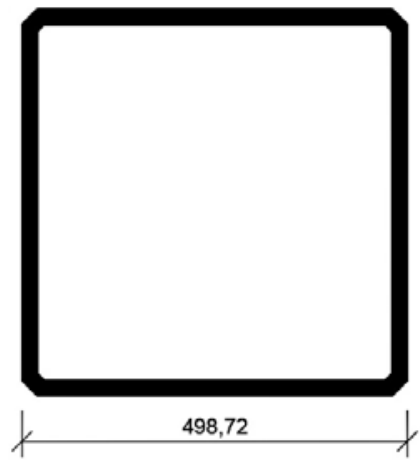

a.1)

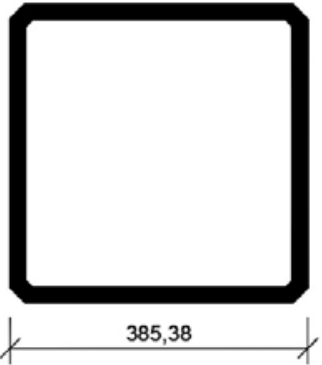

a.2)

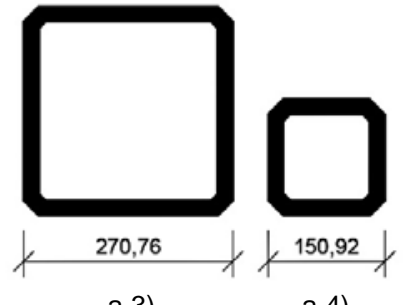

a.3)

a.4)

Fig. 05 Esquemas de manzanas cerradas achaflanadas según cantidad de habitantes. Fuente: Elaboración propia.

b) Manzanas cerradas sin chaflanes:

b.1) 71 hab. $=504,69 \mathrm{~m}$. b.2) 57 hab.= 391,69 m. b.3) 43 hab.= 277,79 m. b.4) 29 hab.= 160,62 $\mathrm{m}$.
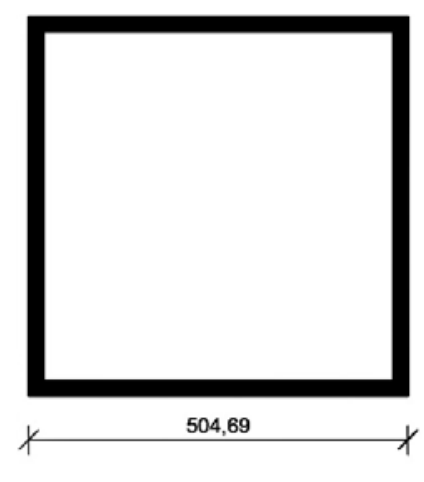

b.1)

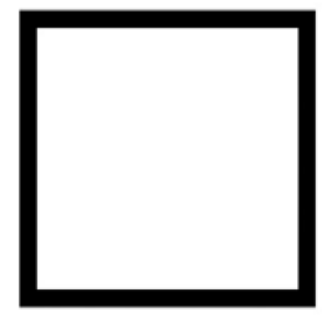

391,69

b.2)

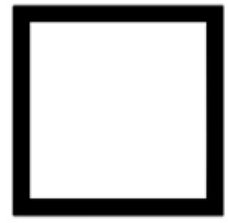

277,79

b.3)

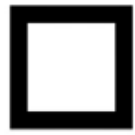

$\varlimsup^{160,62} \Varangle$

b.4)

Fig. 06 Esquemas de manzanas cerradas sin chaflanes según cantidad de habitantes.

Fuente: Elaboración propia.

c) Manzanas abiertas achaflanadas

c.1) 71 hab.= 229,90 m. c.2) 57 hab.= 172,46 m. c.3) 43 hab.= 113,28 $\mathbf{m}$. c.4) 29 hab.= sin resultado.

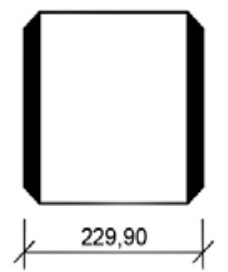

C.1)

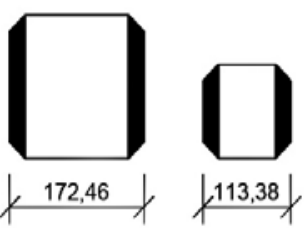

C.2)

c.3)

C.4)

Fig. 07 Esquemas de manzanas abiertas achaflanadas según cantidad de habitantes.

Fuente: Elaboración propia. 
d) Manzanas abiertas sin chaflanes:

d.1) 71 hab.= 242,34 m. d.2) 57 hab.= 185,84 m. d.3) 43 hab.= 128,81 m. d.4) 29 hab. $=70,31 \mathrm{~m}$.

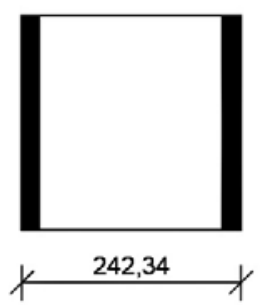

d.1)

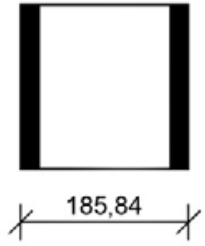

d.2)

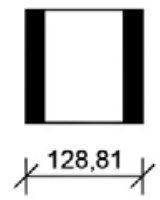

d.3)

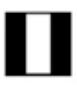

70,31

d.4)

Fig. 08 Esquemas de manzanas cerradas sin chaflanes según cantidad de habitantes. Fuente: Elaboración propia.

En base a estos resultados, Cerdà concluye: "Entre los diversos valores que resultan de la construccion de las fórmulas ¿cual es el que deberemos adoptar? En nuestro concepto es el que nos dá para la manzana 113 metros de lado." (1991: 420, §1498). Aunque justifica la elección en base a varios criterios, el que parece tener más peso es el que corresponde a la comodidad de los transeúntes y a la economía de la ciudad "Porque no es excesiva para la comodidad de los transeúntes de distancia entre calle y calle, ni ecsagerado el número de estas y el de alcantarillas para que su conservación pueda regularse de gravosa para la municipalidad" (1991: 420, §1502).

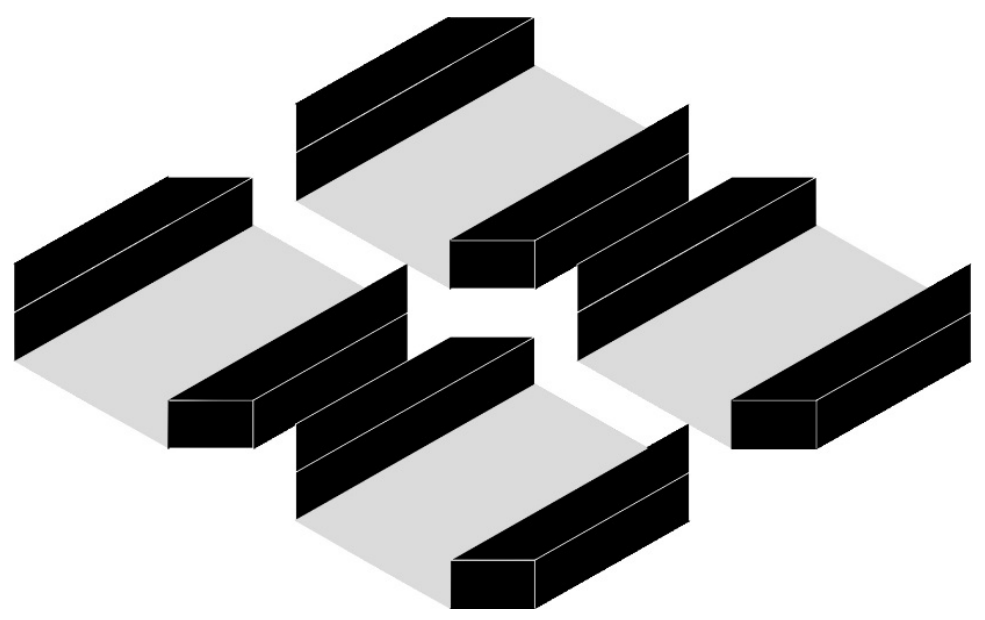

Fig. 09 Isometría del modelo de manzana escogida por Cerdà.

Fuente: Elaboración propia.

La medida seleccionada sospechosamente es la más cercana a la de las referencias sudamericanas -Lima 110 metros y Buenos Aires 116 metros, justo el punto medio-. Se puede inferir por tanto que Cerdà escogió aquella que más se parecía a los ejemplos americanos e incluso pareciera que Cerdà justifica la elección de un punto de llegada seleccionado previamente (De Terán, 1999) a partir de la implementación de una metodología operativa. 


\section{Influencia}

Se esboza así la referencia del "modelo clásico" en el proyecto del Ensanche de Barcelona y en el propio pensamiento de Cerdà, influencia que es explicita en lo que se refiere a forma, orden, ocupación del espacio, y capacidad de expansión -aspecto que Cerdà valora mucho-, e implícita -pero evidente- en cuanto al ancho de las calles y el tamaño de las manzanas.

Ya habiendo ilustrado la influencia e interpretando como se manifiesta, queda entonces aclarar la naturaleza de dicha referencia. Al respecto, se puede decir que existen algunas diferencias entre lo que Cerdà conoce o piensa de la ciudad hispanoamericana -aquellas de esquema cuadricular- y lo que gracias a estudios posteriores se ha logrado determinar sobre esta.

En primer lugar, las medidas que indica Cerdà sobre la ciudad de Lima y la de Buenos Aires no corresponden exactamente ni con los modelos teóricos (García Fernández, 1987) -aquellos provenientes a las referencias históricas-, ni con las medidas actuales -según cartografía-.

\begin{tabular}{|c|c|c|c|}
\cline { 2 - 4 } \multicolumn{1}{c|}{} & Modelos teóricos & Actuales* & Cerdà (TCC) \\
\hline Lima & $\begin{array}{c}\text { Manzanas de } 125,40 \mathrm{~m} . \\
\text { Ancho de calle } 11,10 \mathrm{~m} .\end{array}$ & $\begin{array}{c}\text { Manzanas de } 116,00 \mathrm{~m} . \\
\text { Ancho de calle } 10,50-15,00 \mathrm{~m} .\end{array}$ & $\begin{array}{c}\text { Manzanas de } 110,00 \mathrm{~m} . \\
\text { Ancho de calle } 15,00-20,00 \mathrm{~m} .\end{array}$ \\
\hline Buenos Aires & $\begin{array}{l}\text { Manzanas de } 117,00 \mathrm{~m} . \\
\text { Ancho de calle } 9,20 \mathrm{~m} .\end{array}$ & Manzanas de $117,00 \mathrm{~m}$. & Manzanas de $116,00 \mathrm{~m}$. \\
& \multicolumn{2}{|c|}{ Ancho de calle $9,20-11,00 \mathrm{~m} .{ }^{* *}$} & Ancho de calle $20,00 \mathrm{~m}$. \\
\hline
\end{tabular}

Tabla 01 Comparación entre los largos de manzana y anchos de calle de los modelos teóricos, actuales y planteadas por Cerdà de Lima y Buenos Aires.

Fuente: Elaboración propia a partir de García Fernández (1987) y de cartografía recuperada en línea de: https://www.openstreetmap.org

La referencia de Cerdà de Lima le da un tamaño de manzana menor del que corresponde al modelo teórico y a las medidas actuales, pero lo que dista más es el ancho de la calle, que es sensiblemente mayor según Cerdà. En cuanto a Buenos Aires, sorprende la similitud entre el modelo teórico, la medida actual, y la de Cerdà -solo menor un metro- para el lado de las manzanas. Sin embargo, la medida del ancho de las calles es mucho mayor en la referencia de Cerdà, mientras que en los otros dos es similar. El ancho de la calle en ambos casos es mucho más grande para Cerdà de lo que se conoce de estas ciudades.

Se descarta la posibilidad de que las medidas de Cerdà correspondan a estados previos a los actuales, pues no se encuentran referencias validas que lo ratifiquen, y la tendencia en los cascos históricos de estas urbes como sucede en Buenos Aires- es al contrario, generalmente han ocurrido ampliaciones de las calles, no del tamaño de la manzana. No obstante, en el plano de Buenos Aires de Philippe Bertrès de $1822 \mathrm{y}$ en el plano de la misma ciudad de César Bacle de 1830 puede observarse una proporción exagerada del tamaño de la calle, y dado que estos planos son anteriores pero relativamente contemporáneos al Plan Cerdà es posible -aunque no comprobado-que hayan servido de referencia al autor del Ensanche e influenciaran su estudio del caso de Buenos Aires -concretamente en la percepción errada del tamaño de la calle, 20 metros- 


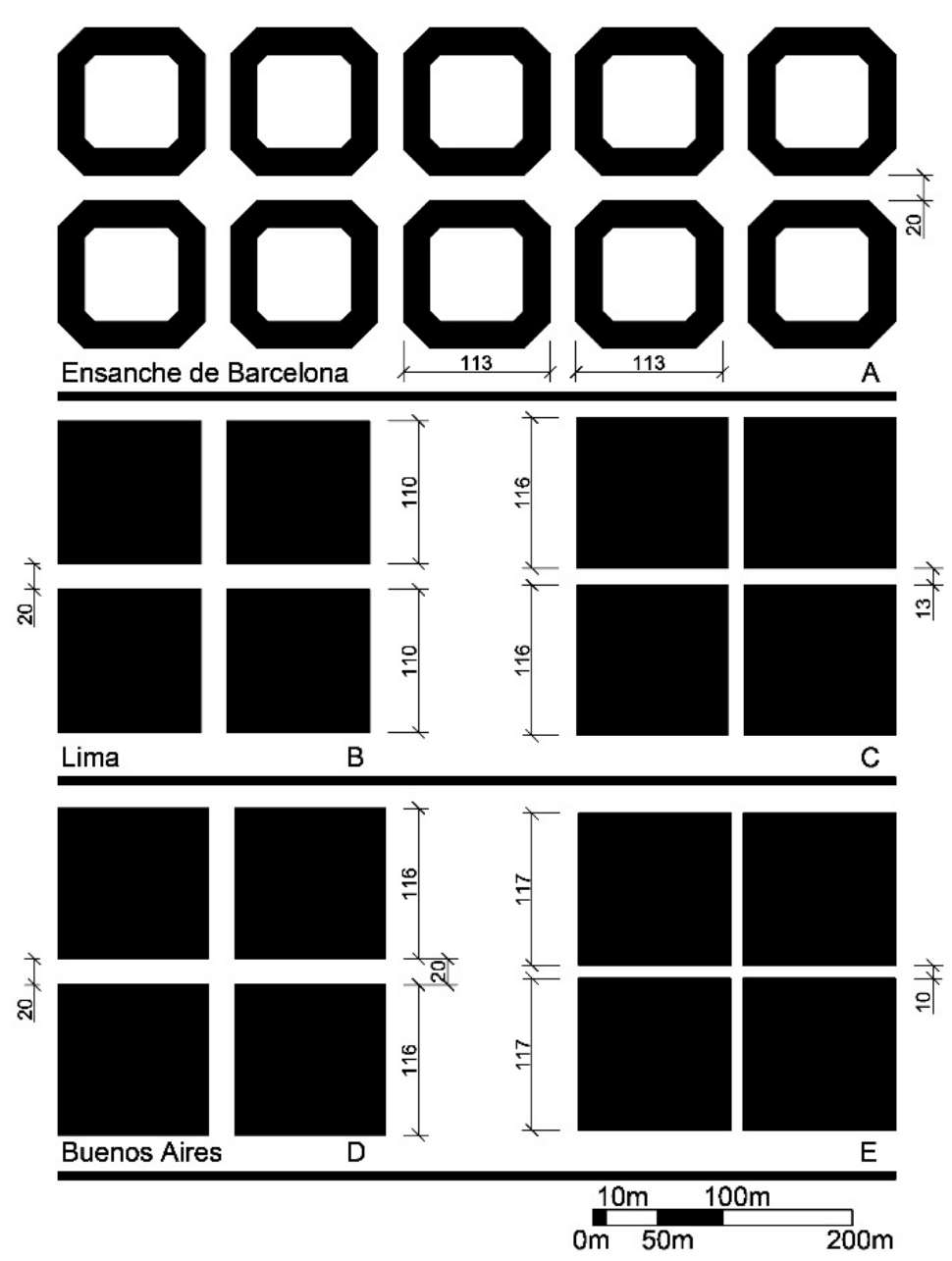

Fig. 10 Manzanas comparadas: A) Plan Cerdà, B) Lima según Cerdà, C) Lima según cartografía, D) Buenos Aires según Cerdà, E) Buenos Aires según cartografía

Fuente: Elaboración propia.

Pero más importante que las diferencias dimensionales son las diferencias conceptuales entre los modelos. El tamaño de las manzanas, que es el módulo esencial en el Ensanche de Barcelona (Soria, Puig, 1999), medida en la que Cerdà más se fija y para la cual realiza mayor sistematización para determinarla, no es el módulo que genera las medidas de la ciudad en el "modelo clásico", sino la Plaza Mayor de cada una de las ciudades, a partir de la cual se genera, por extensión del trazado de las calles que la bordean, las manzanas que componen la ciudad -especialmente las ocho que rodean a la plaza en el esquema cuadricular-. Por esto, la medida de la plaza establece el resto de las medidas. "Así, el núcleo esencial es la plaza, corazón y centro de la urbe, que da el módulo y medida de la totalidad..." (Bonet Correa, 1977: 685).

En relación al tamaño de este módulo, en Hispanoamérica se evidencia una gran variación dimensional - hecho constatable tanto cartográficamente como con aerofotografía-, probablemente con más de 50 metros de variación - de lado- entre las plazas mayores más pequeñas y las más grandes, lo que indica que no parece existir una medida estándar y que por tanto el modelo cuadricular hispanoamericano es un esquema geométrico sin escala determinada. Por tanto, tenemos que aunque Cerdà si trata de razonar el porqué de un tamaño de manzana, en una búsqueda operativa y sistemática, en el contexto hispanoamericano colonial la regularización de este módulo no parece ser tan importante. 
Quizás la diferencia conceptual mayor radica en la interpretación de que es un sistema no jerarquizado debido a la repetición de elementos iguales, interpretación que se infiere pero que no es totalmente explicita. En realidad, la ciudad colonial arquetípica, aunque ciertamente se compone muchas veces de elementos repetidos, es un sistema muy jerarquizado, cuyo núcleo es la plaza mayor, punto centrípeto y centrífugo a partir del cual crecía, se articulaba, y funcionaba la ciudad, y que concentraba los principales poderes de la urbe en él. En esta afirmación sobre la ciudad hispanoamericana coinciden tanto De Terán (1989) -en El sueño de un orden- como Aguilera Rojas (1994) -en Fundación de ciudades hispanoamericanas-.

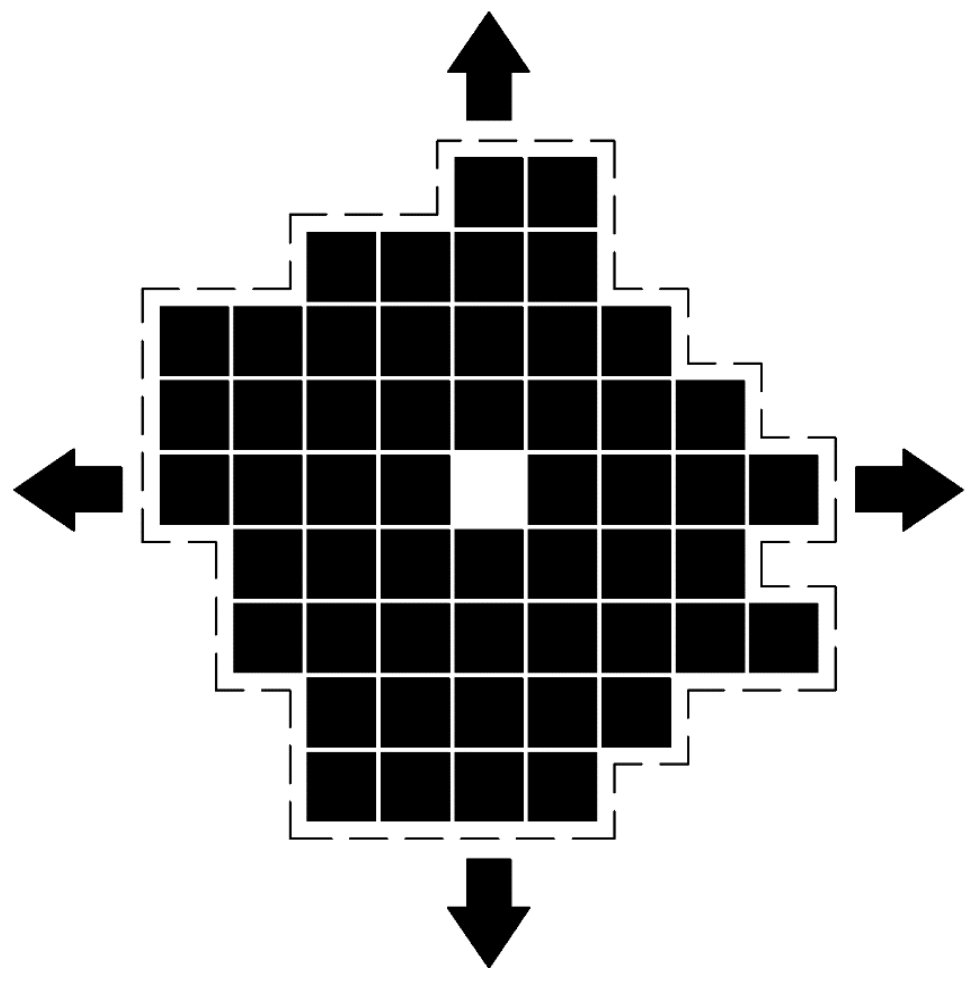

Fig. 11 Modelo de ciudad hispanoamericana expansiva:

Fuente: Elaboración propia.

No obstante, a pesar de existir ciertas diferencias dimensionales y conceptuales, la influencia de las ciudades hispanoamericanas en el Plan de Ensanche de Barcelona es clara, contundente, y sobretodo, estructural. Más allá de un diseño formal específico, en ambos casos se manifiesta el "deseo de un orden", eminentemente práctico y simbólico en el caso hispanoamericano, y con la adición de un componente científico y racionalizador en el caso del Plan Cerdà. Este orden se encuentra además acompañado de una capacidad de expansión en la que puede mantenerse su esquema compositivo básico con la repetición de los elementos, calles y manzanas, "vías" e "intervías" -como denomina el propio Cerdà-. 


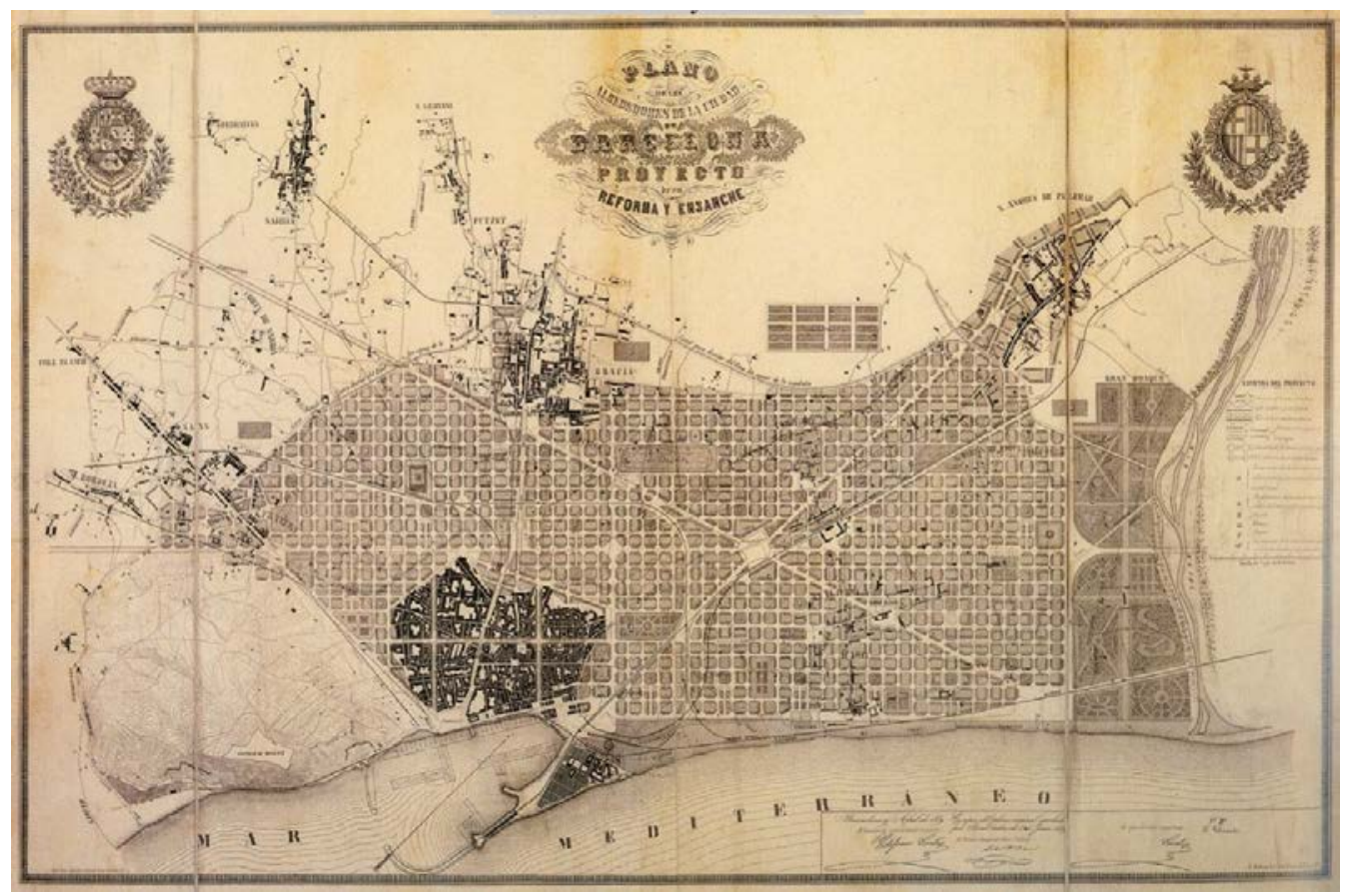

Fig. 12 Plan de Ensanche de Barcelona.

Fuente: Cerdà, I. (1859) Plano de los alrededores de la ciudad de Barcelona y Proyecto de su reforma y ensanche [Plano]. Escala 1:5.000 (GRAU y MONTANER ,2014). Recuperado de: https://upload.wikimedia.org/wikipedia/commons/8/89/PlaCerda1859b.jpg. Consultado 15 de Julio 2019.

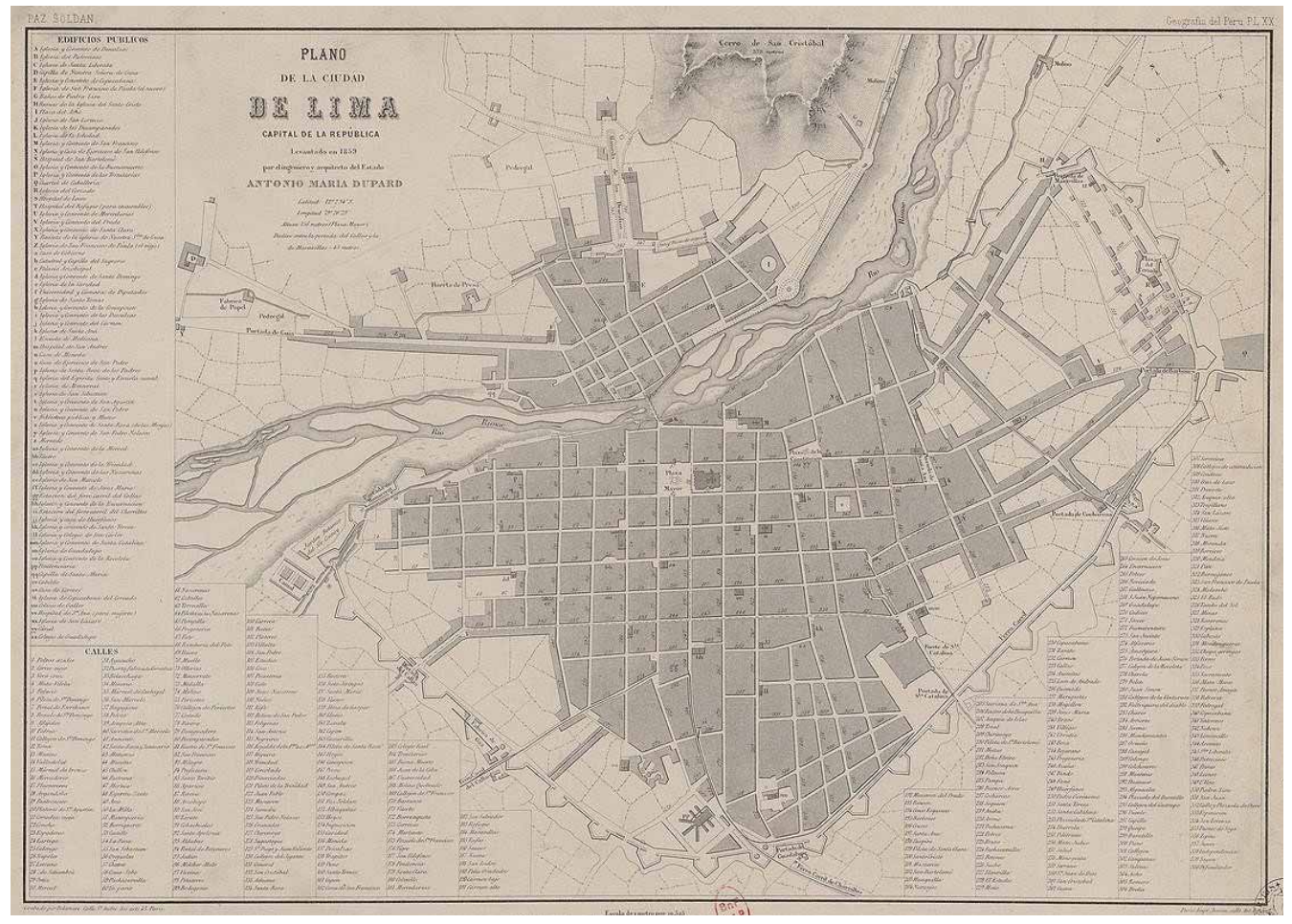

Fig. 13 Plano de Lima 1859.

Fuente: Dupard, A. M. (1859) Plano de la ciudad de Lima. [Plano].

Escala 1:10.000.

Recuperado de: https://gallica.bnf.fr/ark:/12148/btv1b53081335n.

Consultado 15 de Julio 2019. 


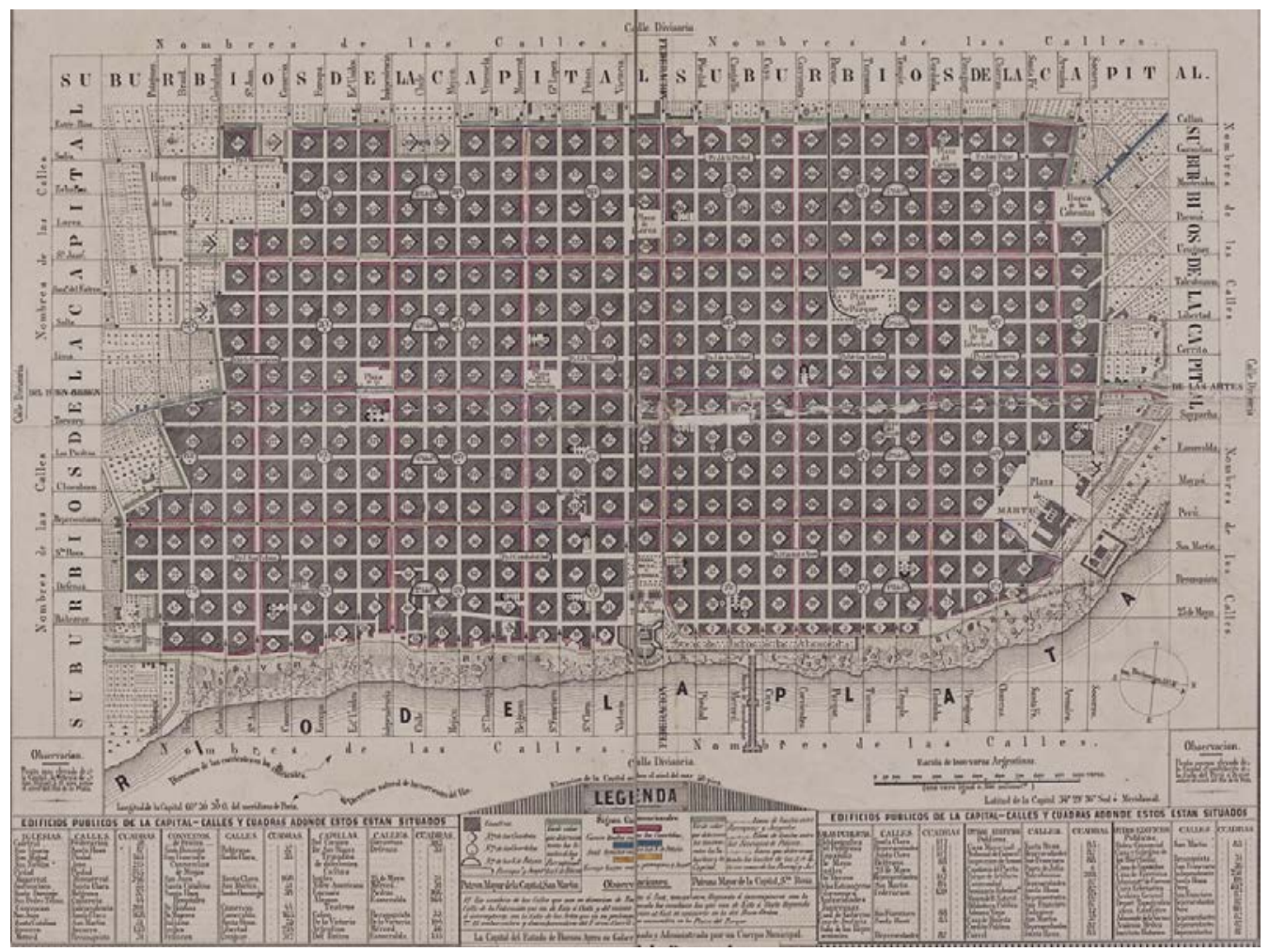

Fig. 14 Plano de Buenos Aires 1856.

Fuente: Grondona, N. (1856) Plano administrativo de la Capital del Estado Argentino de Buenos Ayres. [Plano]. Escala ca. 1:15.200.

Recuperado de: https://catalogo.bn.gov.ar/F/?func=direct\&doc_number=001330694\&local_base=BNA01\#ex1. Consultado 15 de Julio 2019.

\section{Valoración del Proyecto de Ensanche de Barcelona y del pensamiento de Cerdà}

El hecho de que el llamado Plan Cerdà tenga referencias a un modelo previo de ciudad implementado y desarrollado en América no le quita merito, en incluso no pone en peligro la amplia valoración que a lo largo de los años han tenido historiadores e investigadores. Por ejemplo, para El Haddad (2017) el proyecto es producto de un profundo análisis que intenta incorporar un método científico al diseño de la ciudad y sus componentes. En el caso de las conexiones, el cruce de las vías es el aspecto más característico del plan Cerdà para Manuel de Sola-Morales (1978), representando un avance muy interesante y beneficioso para la circulación con respecto al modelo cuadricular, por mencionar solo uno de los elementos más representativos. Y finalmente, en relación con el diseño de la manzana del Plan Cerdà, Serratosa (1995) dice lo siguiente:

"Las dimensiones superficiales de la manzana, la altura reguladora de los edificios, la profundidad edificable, el patio interno de la manzana, la fachada mínima o la relación de la altura máxima con la anchura de la calle son aciertos insuperables que, pese a las oscilaciones (degradación), han permitido conservar hasta hoy la supremacía en el ranking de calidad." (Serratosa, 1995: 47).

Razones sobran para que el Proyecto de Ensanche de Barcelona pueda ser considerado una impresionante referencia de desarrollo urbano, aún en nuestros días. 


\section{Conclusión}

Entendemos entonces que el modelo de la ciudad colonial hispanoamericana es una de las referencias en el Proyecto de Ensanche de Barcelona -con cierta distorsión dimensional y conceptual-, que no se evidencia solo superficialmente, sino que dicha afirmación se fundamenta en documentos del propio autor. Cerdà no solo conoce el modelo, sino que lo alaba y estudia para, en el marco de un análisis sistemático, adaptarlo y aplicarlo en su proyecto, no sin antes hacer las comprobaciones que considera necesarias a partir de las fórmulas expuestas anteriormente.

Por otra parte, es posible entender la influencia del modelo de ciudad hispanoamericana en el Plan Cerdà no como una referencia formal lejana, sino más bien como una realidad cultural cercana a su autor, quien tuvo el interés de estudiarla, y la suficiente lucidez como para comprenderla, nutrirse de ella, y aplicarla finalmente en la Península Ibérica.

Se concluye entonces que el Proyecto de Ensanche de Barcelona se basa en parte en el "modelo clásico" de las ciudades hispanoamericanas, y se presenta por tanto como la continuación y mejora de un sistema de dominio del territorio propio de la cultura hispánica.

\section{BIBLIOGRAFÍA}

AgUILERA RojAs, J. (1994). Fundación de ciudades hispanoamericanas. Madrid: MAPFRE.

Alarcón, M. (2002). El Plan Cerdá para Barcelona y el Urbanismo Argentino del Siglo XIX. En Área Digital, número 2. Recuperado de: https://studylib.es/doc/5546955/el-plan-cerd\%C3\%A1-para-barcelona-y-elurbanismo-argentino-del...

BENÉVOLO, L. (1968). Las nuevas ciudades fundadas en el siglo XVI en la América Latina. Una experiencia decisiva para la Historia de la Cultura Arquitectónica del Cinquecento. En Boletín del Centro de Investigaciones Históricas y Estéticas de la Universidad Central de Venezuela, 9, 117-136.

Bonet CorRea (1977). Ildefonso Cerdá y el urbanismo en Hispanoamérica. En Revista de Indias, 149-150, 679-692.

CERDÀ, I. (1991). Teoría de la construcción de las ciudades aplicada al proyecto de reforma y ensanche de Barcelona - Cerdá y Barcelona. Publicación de los manuscritos inéditos de Idelfons Cerdá sobre el Plan del Ensanche de Barcelona, casi un siglo y medio después de haber sido escritos. Volumen I. Publicado originalmente en 1859. Madrid: Ministerio para las Administraciones Públicas, Ayuntamiento de Madrid, Ayuntamiento de Barcelona.

CERDÀ, I. (1867 - 1968). Teoría general de la urbanización y aplicación de sus principios y doctrinas a la reforma y ensanche de Barcelona. Trabajo ultimado en virtud de Real Autorización de 2 de febrero de 1859, aprobado por Real órden de 7 de junio del mismo año, declarado de utilidad para la enseñanza y de aplicación oficial, por Real decreto de 31 de mayo de 1860, y mandado publicar por Real órden de 20 de diciembre de 1863, á espensas del Estado con fondos especiales votados por las Córtes. Publicado originalmente en 1867. Barcelona: Instituto de Estudios Fiscales. Prólogo a la edición facsímil por Antonio Barrera de Irimo. Estudio sobre "La vida y obra de Ildefonso Cerdá", selección del anexo documental y bibliografía por Fabián Estapé.

El HaddAd, M. (2017). Barcelona: Small-Scale Public Spaces. Tesis doctoral presentada para la defensa del grado de doctor. Universitat de Barcelona. 
GaRcíA FeRnÁndez, J. L. (1987). Análisis dimensional de modelos teóricos ortogonales de las ciudades españolas e hispanoamericanas desde el siglo XII al XIX. En La Ciudad Iberoamericana. Actas del Seminario Buenos Aires 1985 (153-192). Madrid: CEHOPU.

Grau, R. y Montaner, C. (ed.) (2014). Estudis sobre la cartografia de Barcelona, del segle XVIII al XXI. Els mapes d'una ciutat en expansió. Barcelona: Ajuntament de Barcelona, Institut Cartogràfic i Geològic de Catalunya.

HARDoy, J. E. (1972). El modelo clásico de la ciudad Colonial Hispanoamericana. En Actas del XXXVIII Congreso Internacional de Americanistas Stuttgart, 1968, Iv (143-181). München: K. Renner.

Serratosa, A. (1995). El valor del Ensanche de Cerdà, hoy. En Magrinyà, F., Tarragó, S., \& Alacaraz, M. J. (1995) Cerdá, ciudad y territorio. Una visión de futuro. Barcelona: Fundación Catalana per la Recerca.

SolÁ-Morales, M. (1974) Los Ensanches. En SolÀ-Morales, M., Clota, M. D., \& Ordóñez, J. L. G. (1978). Los Ensanches: el Ensanche de Barcelona. Barcelona: Escuela Técnica Superior de Arquitectura. Laboratorio de Urbanismo

Sola-Morales, M. (1978). Querido León ¿Por qué 22 por 22? Arquitectura Bis, $20,7$.

SolÁ-Morales, M. (ed.) (1997) Las formas de crecimiento urbano. Barcelona: Universidad Politécnica de Cataluña.

SORIA, Y., PUIG, A. (1999). Actualidad de la teoría de Cerdá. Ciudad y Territorio. Estudios Territoriales, XXXI:119120, 127-145.

TERÁN, F. de (cord.) (1989). El sueño de un orden. Madrid: CEHOPU.

TERÁN, F. de (1999). El urbanismo europeo en América y el uso de la cuadricula. Ciudad y Territorio. Estudios Territoriales, xxxI:119-120, 21-40.

TERÁN, F. de (2009). Presentación de la edición facsímil del plano de los alrededores de Barcelona y proyecto de su reforma y ensanche, obra de Ildefonso Cerdá (1859). Boletín de la Real Academia de Bellas Artes de San Fernando, 108-10, 211-219. 